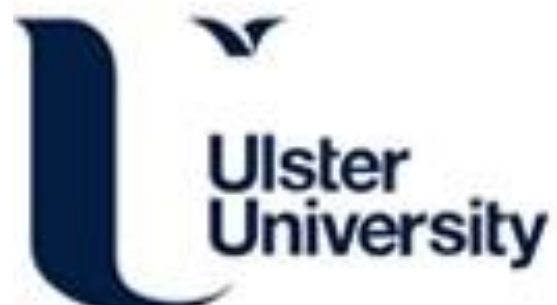

Simulation of Intelligent Computational Models in Biological Systems

Wu, Q., McGinnity, TM., Maguire, LP., Belatreche, A., \& Glackin, B. (2007). Simulation of Intelligent Computational Models in Biological Systems. In Unknown Host Publication (pp. 1974-1978). IEEE. https://doi.org/10.1109//CMLC.2007.4370470

Link to publication record in Ulster University Research Portal

\section{Published in:}

Unknown Host Publication

Publication Status:

Published (in print/issue): 01/08/2007

DOI:

10.1109/ICMLC.2007.4370470

\section{Document Version}

Publisher's PDF, also known as Version of record

\section{General rights}

Copyright for the publications made accessible via Ulster University's Research Portal is retained by the author(s) and / or other copyright owners and it is a condition of accessing these publications that users recognise and abide by the legal requirements associated with these rights.

\section{Take down policy}

The Research Portal is Ulster University's institutional repository that provides access to Ulster's research outputs. Every effort has been made to ensure that content in the Research Portal does not infringe any person's rights, or applicable UK laws. If you discover content in the Research Portal that you believe breaches copyright or violates any law, please contact pure-support@ulster.ac.uk. 


\title{
SIMULATION OF INTELLIGENT COMPUTATIONAL MODELS IN BIOLOGICAL SYSTEMS
}

\author{
QING-XIANG WU, MARTIN MCGINNITY, LIAM MAGUIRE, AMMAR BELATRECHE, \\ BRENDAN GLACKIN
}

\author{
School of Computing and Intelligent Systems, University of Ulster at Magee Derry, BT48 7JL, Northern Ireland, UK \\ E-MAIL: \{q.wu, tm.mcginnity, lp.maguire, a.belatreche, b.glackin\}@ulster.ac.uk
}

\begin{abstract}
:
The human brain can perform a range of complicated computations and logical reasoning using neural networks with a huge number of neurons. Since Hodgkin and Huxley proposed a set of equations to describe the electrophysiological properties of spiking neurons, various network structures of neurons have been developed through neuroscience research that can now be simulated by electronic circuits or computer programs. In this paper, an adaptive learning mechanism is simulated based on the biological property related to the spike time dependent plasticity of synapses. A demonstration shows that such spiking neurons are able to develop their specific receptive field for recognition of patterns. This mechanism can be used to explain some adaptive behaviours in biological systems. It is can also be applied to artificial intelligent systems.
\end{abstract}

\section{Keywords:}

Spiking neural network; Computational model; Adaptive learning; Spiking time dependent plasticity

\section{Introduction}

The term, neuronal circuit, is encountered more and more frequently in neuroscience literature. The local neuronal circuit in the recurrent networks of the human visual cortex has been used to explain the mechanism of context learning [1]. The generation of rhythmic motor behaviours is controlled by neuronal circuits which are called central pattern generators [2]. Recurrent inhibitory loops are simple neuronal circuits found in the central nervous system [3]. By expressing several forms of synaptic plasticity, a single neuron can convey an array of different signals to the neural circuit in which it operates [4]. Scientists working in neuroscience have made significant progress over the past ten years with respect to the physiological and synaptic properties of individual neurons and some specific local neuronal circuits [1-5]. However, there is very limited progress in the understanding of what is the exact architecture of the neural network in the brain and how the network works based on the spiking neuron model [2]. As the electro-physiological properties of biological neurons can be described by differential equations, such spiking neuronal circuits can be simulated by electronic circuits or computer simulation programs. For example, the software NEURON provides an established tool for the simulation of different neuronal circuits. Since it costs very high computational complexity to simulate dynamics and plasticity of biological neurons, biological neural network behaviours are usually simulated using statistic models based on firing rate or instantaneous firing frequencies. In the literature the probability of a neurotransmitter release is regarded as the strength of a synapse [1]. The pre- and post-synaptic instantaneous firing frequencies are used to formulate the function based on nonlinear Hebbian and anti-Hebbian plasticity. However, simulations based on spiking neurons have not been done. The gastric mill and pyloric circuits in the stomatogastric nervous system are very detailed, but they have not been simulated using electronic circuits based on spiking neuron model [2]. Therefore, this paper presents approaches to simulate biological neuronal circuits using electronic circuits or computer programs.

In Section 2, an efficient approach for the simulation of spiking neurons is introduced. Then in Section 3, simulation of spike time dependent plasticity is presented. In Section 4, a spiking neural network for pattern recognition is proposed, and a demonstration is applied to show the principle how the network can develop a specific receptive field based on STDP (spike time dependent plasticity). The neuronal circuits have been simulated by computer software and FPGA circuits. In Section 5 related issues and further work are discussed.

\section{Simulation of Spiking Neurons}

Since the Hodgkin-Huxley neuron model requires very high computational complexity, the conductance based integrate-and-fire model is widely used to spiking neural networks. The firing property of this model is very close to that of the Hodgkin-Huxley model in NEURON software 
[6]. In this integrate-and-fire model, the membrane potential $v(t)$ is governed by the following equations [6-9].

$$
c_{m} \frac{d v(t)}{d t}=g_{l}\left(E_{l}-v(t)\right)+\sum_{j} \frac{w_{j} g_{j}^{s}(t)}{A_{s}}\left(E_{s}-v(t)\right)
$$

where $c_{m}$ is the specific membrane capacitance, $E_{l}$ is the membrane reversal potential, $E_{s}$ is the reversal potential of membrane, $s \in\{i h, e x\}$ ( $i h$ and ex indicate inhibitory and excitatory synapses respectively), $w^{j}$ is a weight for synapse $j$, and $A_{s}$ is the membrane surface area connected to a synapse. If the membrane potential $v$ exceeds the threshold voltage, $v_{t h}$, it is reset to $v_{\text {reset }}$ for a refractory $\tau_{\text {ref }}$ and an action potential event is generated. Figure 1 shows that a neuron receives spike trains from three afferent neurons in a receptive field.

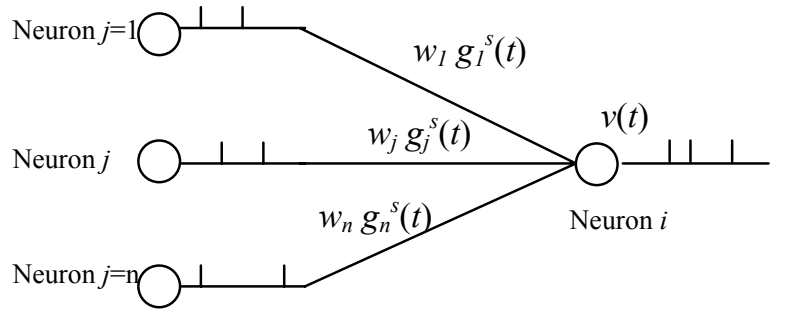

Figure 1. Conductance based synaptic connections in the SNN

The variable $g_{j}^{s}(t)$ represents the conductance of synapse $j$ at time $t$. When an action potential reaches the synapse at $t^{a p}$, the conductance is increased by the following expression.

$$
g_{j}^{s}\left(t^{a p}+t_{j}^{\text {delay }}+d t\right)=g_{j}^{s}\left(t^{a p}+t_{j}^{\text {delay }}\right)+q_{s}
$$

Otherwise, the conductance decays as illustrated in the following equation.

$$
\frac{g_{j}^{s}(t)}{d t}=-\frac{1}{\tau_{s}} g_{j}^{s}(t)
$$

where $q_{s}$ is called the peak conductance (an increment of conductance caused by the action potential). Neuron $i$ integrates the currents from afferent synapses and increases the membrane potential according to Equation (1).

\subsection{Computer Software Simulation}

Equations (1)-(3) can be solved using numerical solutions such as the Euler or Runge-Kutta methods. Let $\Delta t$ represent time step and $t_{1}, t_{2}, \ldots t_{k}$ represent discrete time, i.e. $t_{n+1}=t_{n}+\Delta t$. Using the Euler method, the neuron model is simulated by following program.
1. Set parameters $c_{m}, E_{s}, g_{l}, w_{j}$

2. Set initial values for $v\left(t_{0}\right), g_{j}^{s}\left(t_{0}\right)$

3. Transfer input action potentials or external currents to corresponding peak conductance $q_{s}$.

4. Calculate $g_{j}^{s}(t)$

$$
g_{j}^{s}\left(t_{n+1}\right)=\left\{\begin{array}{lc}
-\frac{1}{\tau_{s}} g_{j}^{s}\left(t_{n}\right) \Delta t+q_{s} & \text { if there is a action potential } \\
-\frac{1}{\tau_{s}} g_{j}^{s}\left(t_{n}\right) \Delta t & \text { or external current. }
\end{array}\right.
$$

5. Calculate $v(t)$

$$
\begin{aligned}
v\left(t_{n+1}\right) & =v\left(t_{n}\right)+\frac{1}{c_{m}}\left[g_{l}\left(E_{l}-v\left(t_{n}\right)\right)+\right. \\
& \left.\sum_{j} \frac{w_{j} g_{j}^{s}\left(t_{n+1}\right)}{A_{s}}\left(E_{s}-v\left(t_{n}\right)\right)\right] d t
\end{aligned}
$$

6 . if $v\left(t_{n+1}\right)>$ threshold then generate a spike and fall into refractory state for a period $\tau_{r e f}$.

7. if $t<t_{\max }$ then $\mathrm{n}=\mathrm{n}+1$ : goto 3

8. end

\subsection{FPGA Circuit Simulation}

Based on the algorithm in Section 2.1, FPGA (Field Programmable Gate Array) hardware circuits can be designed using Simulink and Xilinx system generator. This is very practical approach. Different neuron models can be designed as SNNs (Spiking Neural Network) blocks in the Matlab development environment. Previous work has developed SNN Blocks in the SenseMaker project [10]. For example, Figure 2 shows that an integrate-and-fire neuron block is tested in the high-level design environment.

The block in the middle is an integrate-and-fire neuron model block that is designed using element blocks provided by Xilinx System Generator. In this environment, a lot of tools in Simulink library can be applied to test the circuit design, for example, signal generators and scopes. In Figure 2, a pulse generator and a scope have been applied to test the integrate-and-fire neuron. A poisson spike train generated by Matlab enters the block through the gateway of Xilinx System Generator. The simulation results can be seen in a pop-up window from the scope shown in Figure 3. The bottom panel in Figure 3 shows the input Poisson spike train. The middle panel shows the conductance changes caused by the input Poisson spike train. The top panel shows the membrane potential changes and fires. 


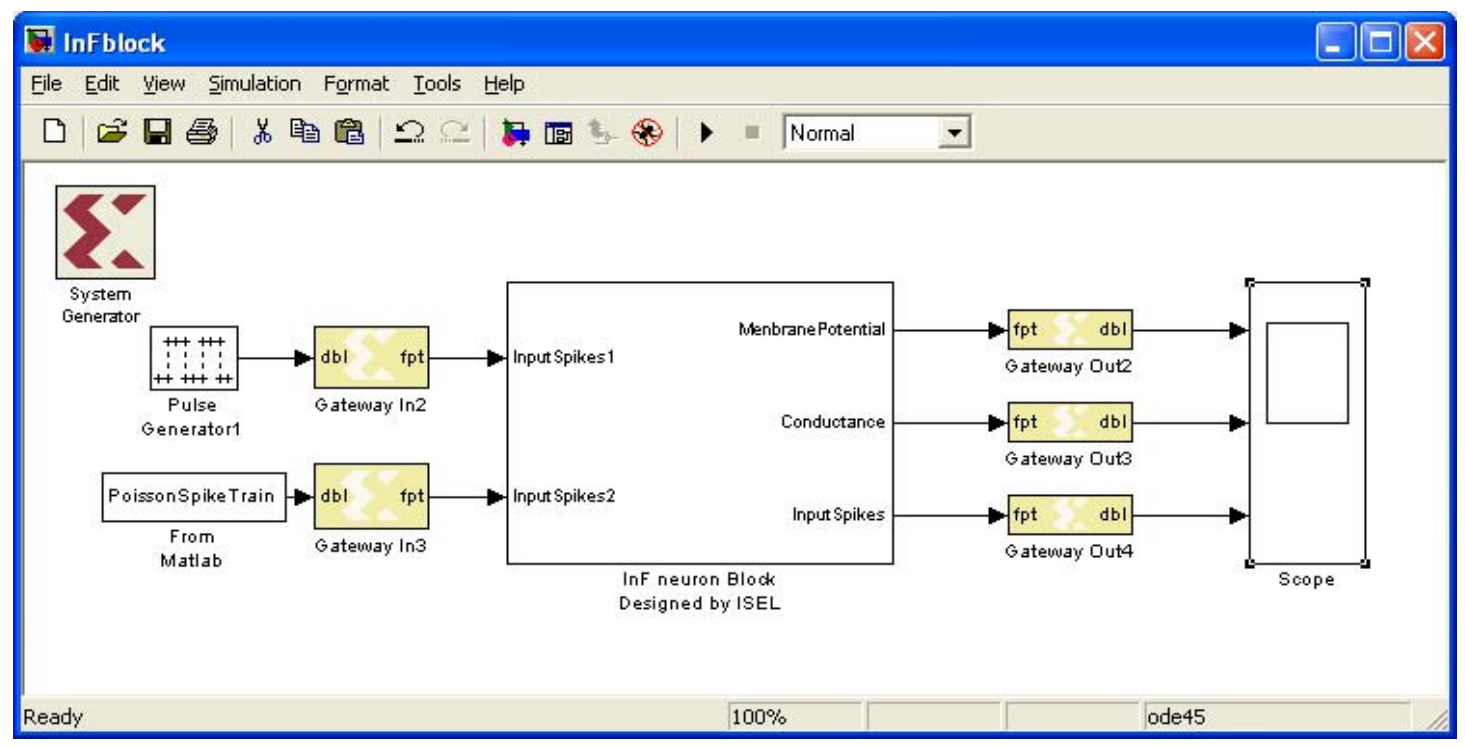

Figure 2. The circuit of Integrate-and-fire neuron model Block

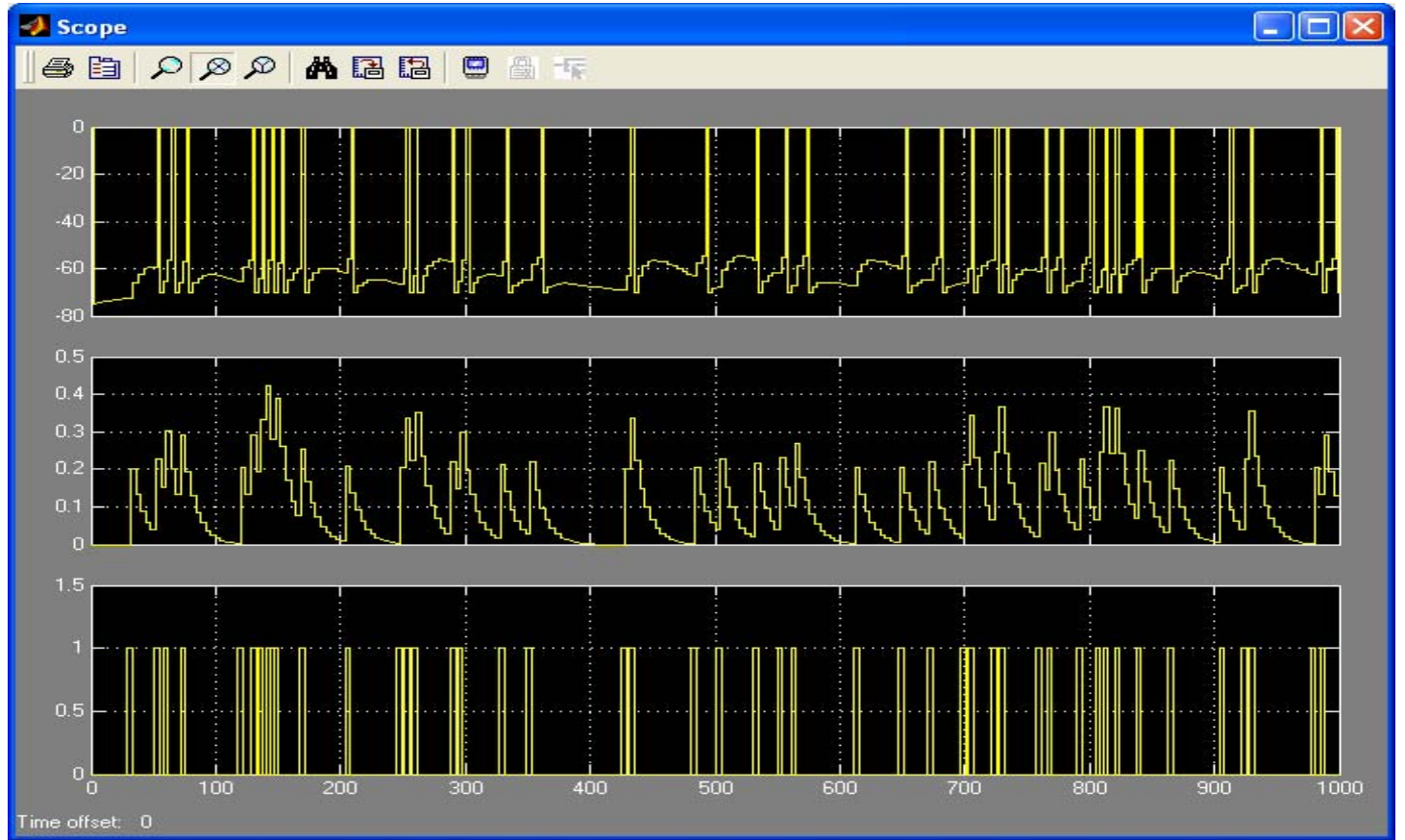

Figure 3. Simulation results of integrate-and-fire neuron Block. The first row shows membrane potential $v(t)$. The second row shows $g_{j}^{s}(t)$. The third row shows input spike train

By using this high level design tool, the neuron models, parameters and network structures can be changed easily. This design can be automatically transferred to HDL
(Hardware Description Language) code and implemented on FPGAs. The design also can be modified in the HDL code level and simulated in Modelsim environment. 


\section{Simulation of Spike Time Dependent Plasticity}

Changes in the synaptic connections between neurons are widely believed to contribute to memory storage and learning. These changes are thought to occur through time correlation-based mechanism such as Hebbian plasticity. Spike Timing-Dependent Plasticity (STDP) was also found in biological neurons [1]. The synaptic plasticity model has been explored based on the fact that a synaptic potentiation and depression can be induced by precisely timed pairs of synaptic events and postsynaptic spikes.

In order to simulate STDP learning in a spiking neural network, the implementation approach proposed in [11-12] is employed. Each synapse in an SNN is characterized by a peak conductance qs (the peak value of the synaptic conductance following a single presynaptic action potential) that is constrained to lie between 0 and a maximum value qs_max. Every pair of pre- and postsynaptic spikes can potentially modify the value of qs, and the changes due to each spike pair are continually summed to determine how qs changes over time. The simplifying assumption is that the modifications produced by individual spike pairs combine linearly.

A presynaptic spike occurring at time tpre and a postsynaptic spike at time tpost modify the corresponding synaptic conductance by

where $\Delta t=t_{\text {post }}-t_{\text {pre }}$ and

$$
q_{s} \rightarrow q_{s}+q_{s \_ \text {max }} F(\Delta t)
$$

$$
F(\Delta t)=\left\{\begin{array}{c}
A_{+} \exp \left(\Delta t / \tau_{+}\right), \text {if } \Delta t>0 \\
-A_{-} \exp \left(\Delta t / \tau_{-}\right), \text {if } \Delta t \leq 0
\end{array}\right.
$$

The time constants $\tau_{+}$and $\tau_{\text {- determine the ranges of }}$ pre- to postsynaptic spike intervals over which synaptic strengthening and weakening are significant, and $\mathrm{A}_{+}$and $\mathrm{A}_{-}$ determine the maximum amount of synaptic modification in each case. The experimental results indicate a value of $\tau_{+}$in the range of tens of milliseconds (about $20 \mathrm{~ms}$ ). The parameters for STDP in our experiments are as follows; $q_{s_{-} \max }=0.01, A_{+}=0.01, A_{-}=0.005, \tau_{+}=20 \mathrm{~ms}, \tau_{-}=100 \mathrm{~ms}$. The function $F(\Delta t)$ for synaptic modification is shown in Figure 4. It is clear that $q_{s}$ is not a constant. It is a variable dependent on pre- and post- synaptic spikes which makes an SNN a dynamic network.

\section{Spiking Neural Network for Pattern Recognition}

Training can be used to improve the ability of humans for detection of small changes in a variety of visual attributes such as position, orientation, motion, depth, spatial phase, hyper-acuity and texture segmentation [1]. In order to simulate this mechanism, an SNN based STDP is proposed to explain how a spiking neuron can develop its receptive field with an adaptive learning mechanism. The architecture of the SNN is shown in Figure 5.

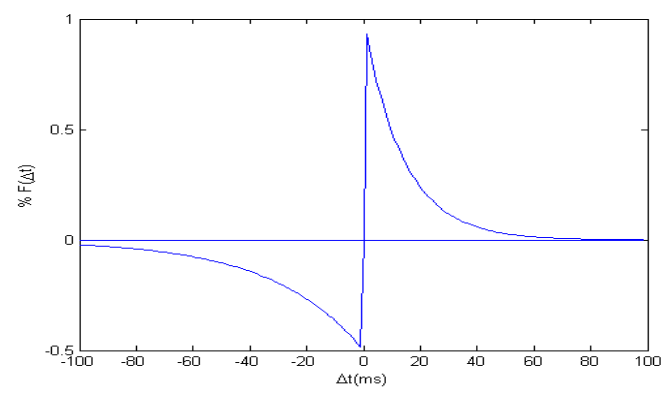

Figure 4. Synaptic modification

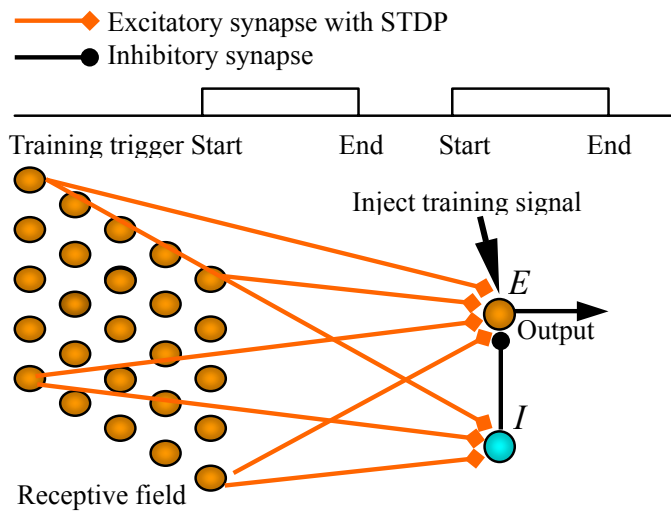

Figure 5. An Architecture of STDP-based SNN

As shown in Figure 5, neuron $I$ is fully connected to neurons in the receptive field through inhibitory synapses. Neuron $E$ is fully connected to neurons in the receptive field through excitatory synapses and it is also connected to neuron $I$ through an inhibitory synapses. Suppose that all the inhibitory synapses have a constant strength and all the excitatory synapses have a randomly weak strength initially. Since the weights of the excitatory synapse are a random distribution and neuron $I$ inhibits neuron $E$, neuron $E$ is very difficult to fire initially. After a training process based on STDP learning rule, neuron $E$ can be trained to respond to specific pattern in the receptive field. The formal training procedure can be done using following steps.

Step 1: An target pattern is presented in the receptive field at meanwhile an external current (from the training signal shown in Figure 5) is injected into neuron $E$ and makes the neuron $E$ fires relative to the pattern. According to STDP rule, the corresponding weights of the excitatory synapses will get either potentiated or depressed.

Step 2: the target pattern is removed and a random plot pattern is presented in the receptive field at meanwhile the external current is cancelled. Neuron $E$ fires without correlation 
to the pattern. The corresponding weights of some excitatory synapses can be depressed.

Repeating the two steps, neuron $E$ can be trained to respond to the target pattern. For example, a $5 \times 5$ pattern as shown in Figure 6(a) is used as the target pattern. Figure 6(b) and Figure $6(\mathrm{c})$ are examples of non-target patterns generated by random plot.

After training, a vertical orientation receptive field is obtained. The membrane potential of neuron $E$ in response to different input patterns are shown in Figure 7.

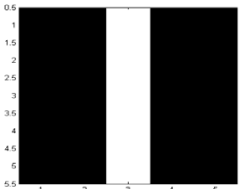

(a)

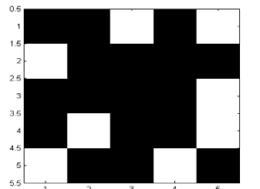

(b)

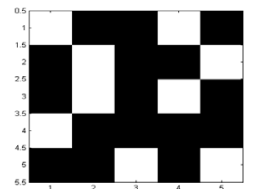

(c)

Figure 6. Training patterns
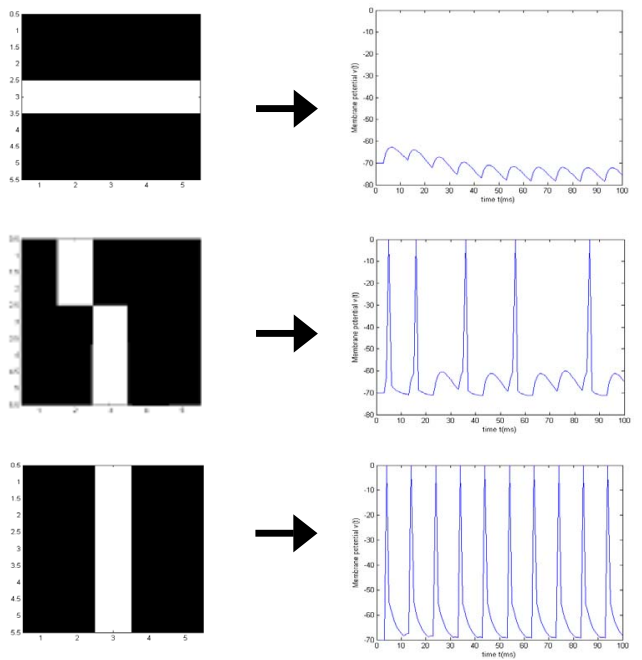

Input patterns

Membrane potential
Figure 7. Neuron $E$ responds to different patterns.

\section{Discussion and Further Work}

In this paper a STPD based learning mechanism is presented. Learning process for a vertical orientation selectivity receptive field is used to demonstrate this mechanism. This network model is inspired by biological principles, and it is implemented in software and FPGA hardware. It has potential for application in artificial intelligent systems. This paper only gave a very simple example for this biologically inspired spiking neural network. It is clear that there is a potential to apply it to more complicated tasks such as face recognition, image classification and object recognition. These are topics for further study.

\section{Acknowledgements}

The authors acknowledge the financial and technical contribution of the SenseMaker project (IST-2001-34712), which is funded by the EC under the FET life like perception initiative.

\section{References}

[1] Yael Adini, Dov Sagi, and Misha Tsodyks, "Context-enabled learning in the human visual system”, Nature, Vol 415, pp.790 - 793, 07 Feb 2002.

[2] Michael P. Nusbaum, and Mark P. Beenhakker, "A small-systems approach to motor pattern generation", Nature, 417, pp.343 - 350 , 16 May 2002.

[3] Fre'de' ric Pouille, Massimo Scanziani, "Routing of spike series by dynamic circuits in the hippocampus", Nature, Vol 429, pp.717 - 723, 30 May 2004.

[4] L. F. Abbott, and Wade G. Regehr, "Synaptic computation", Nature, Vol 431, pp.796 - 803, 13 Oct 2004.

[5] Alison Abbott, Neuroscience Deep in thought, Nature, Vol 436, pp.18 - 19, 06 Jul 2005.

[6] E. Müller, "Simulation of High-Conductance States in Cortical Neural Networks", Masters thesis, University of Heidelberg, HD-KIP-03-22, 2003.

[7] A. Destexhe, E. Marder, "Plasticity in single neuron and circuit computations", Nature, Vol 431,pp.789795, 2004.

[8] Q. X. Wu, T. M. McGinnity, L. P. Maguire, B. Glackin and A. Belatreche, "Learning Mechanism in Networks of spiking Neurons", Studies in Computational Intelligence, Springer- Verlag, Vol 35, pp.171-197, 2006.

[9] Q. X. Wu, T. M. McGinnity, L. P. Maguire, A. Belatreche and B. Glackin, "Adaptive Co-Ordinate Transformation Based on Spike Timing-Dependent Plasticity Learning Paradigm", LNCS, Springer, Vol 3610, pp.420-429, 2005.

[10] SenseMaker Project (IST-2001-34712) funded by the European Union under the "Information Society Technologies" Programme, 2002-2006.

[11] S. Song, and L.F. Abbott, "Column and Map Development and Cortical Re-Mapping Through Spike-Timing Dependent Plasticity", Neuron, vol 32, pp.339-350, 2001.

[12] Joseph E. Atkins, Robert A. Jacobs, and David C. Knill, "Experience-dependent visual cue recalibration based on discrepancies between visual and haptic percepts", Vision Research, Vol 43, No. 25, pp.2603-2613, 2003. 